\section{Intramural tracking in esophageal pseudodiverticulosis}

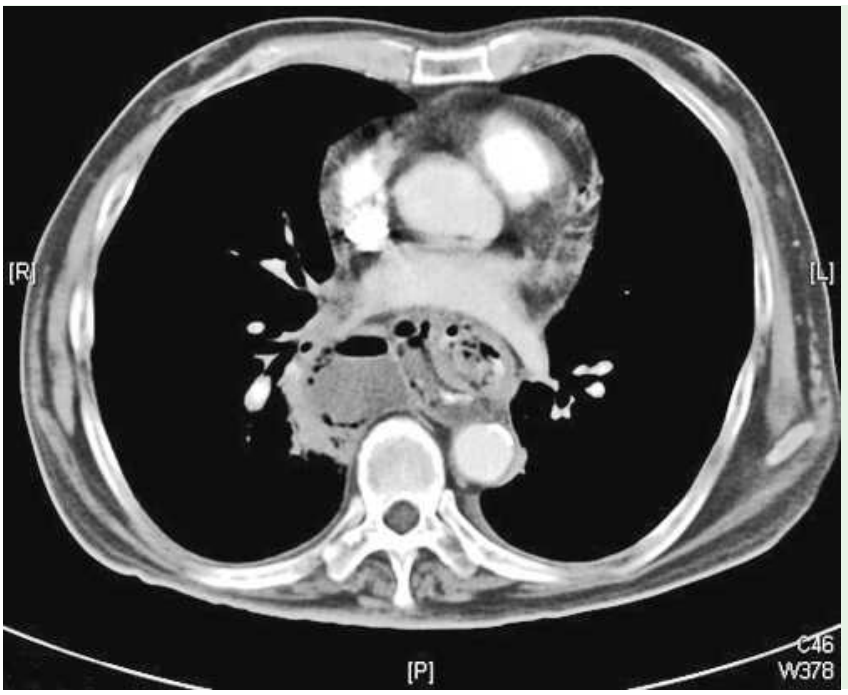

Fig. 1 Computed tomography (CT) of the thorax in July 2004 showing paraesophafluid levels.

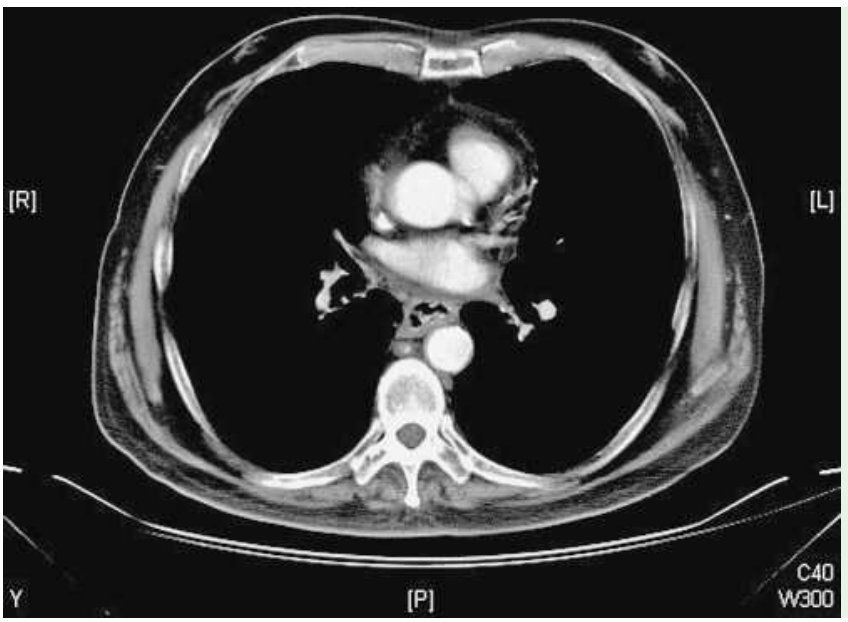

A 60-year-old man with diabetes mellitus and a long history of alcohol addiction presented with dysphagia and regurgitation of food. An initial endoscopy showed ulcerated lesions, which only yielded inflammatory cells on multiple biopsies, with no signs of malignancy. He subsequently developed a fever, and computed tomography (CT) of the thorax showed mediastinal abscesses and paraesophageal collections ( Fig. 1 ). Biopsies grew Candida glabrata, and cultures grew Enterobacter and Klebsiella species. The patient responded well to intravenous imipenem.

A repeat endoscopy post-recovery showed multiple ostia throughout his esophageal mucosa, which may have communicated with the abscesses, thus geal collections with

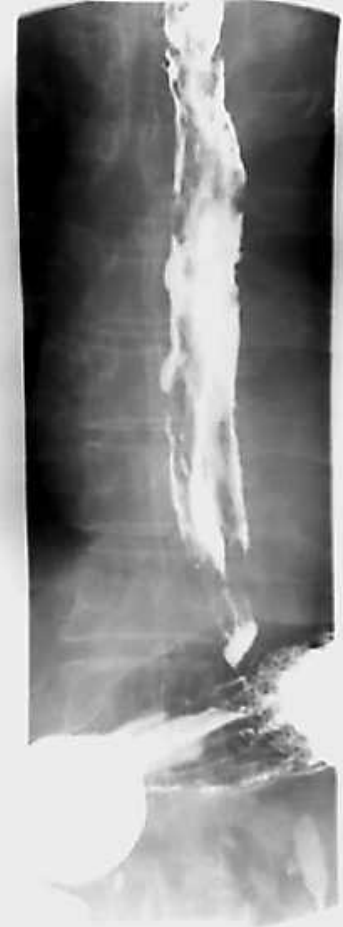

Fig. 3 Barium esophagram in June 2006 showing intramural tracking sinuses running parallel to actual esophageal lumen.

Fig. 2 CT thorax in June 2006 showing resolution of abscesses and paraesophageal collections, but with irregularity of esophageal mucosa.

allowing self-drainage and contributing to the resolution of the infection. A CT thorax 2 years later showed resolution of the collections ( $\bullet$ Fig. 2 ), with remnant irregularities of the esophageal wall. These were illustrated on a barium esophagram to be intramural sinuses dissecting the esophageal wall ( Fig. 3) These sinuses are likely to be ectatic changes of baseline esophageal intramural pseudodiverticulosis, due to chronic infection and inflammation. On endoscopy, some of these sinuses were seen to open into the gastric cardia ( $\bullet$ Fig. 4). Intramural tracking in esophageal intramural pseudodiverticulosis has been found to have a prevalence of up to $50 \%$ in patients with this rare condition, though not as florid as we have described

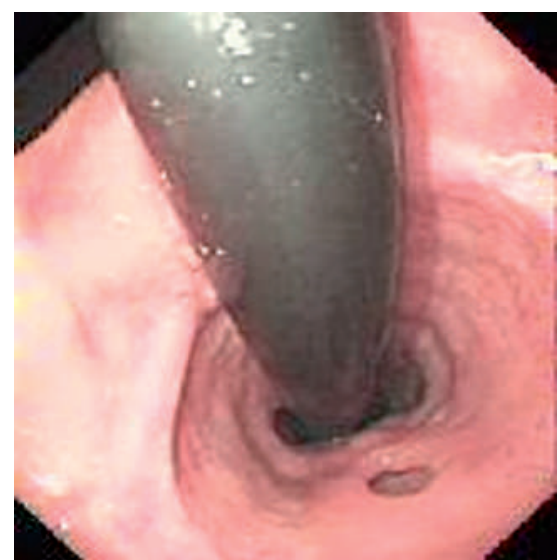

Fig. 4 Endoscope looking back at the gastroesophageal junction, with opening of a sinus track next to it.

[1]. The etiology of esophageal pseudodiverticulosis is unknown, but there have been suggestions of malignant potential [2]. As such, management of our patient includes frequent surveillance with endoscopy, keeping in mind the bizarre architecture of his esophagus and the possibility of malignancy within sinus tracts. The residual symptom of intermittent dysphagia can be treated symptomatically with dilatation during endoscopy [3].

Endoscopy_UCTN_Code_CCL_1AB_2AC_3AF 


\section{E. Wang ${ }^{1}$, A. Tang ${ }^{1}$, S. Venkatesh' ${ }^{2}$, J. So ${ }^{1}$}

${ }^{1}$ Department of General Surgery,

National University Hospital of

Singapore, Singapore

2 Department of Diagnostic Imaging, National University Hospital of Singapore, Singapore

\section{References}

1 Canon CL, Levine MS, Cherukuri R et al. Intramural tracking: a feature of esophageal intramural pseudodiverticulosis. Am J Roentgenol 2000; 175: 371 - 374

2 Plavsic BM, Chen MYM, Gelfand DW et al. Intramural pseudodiverticulosis of the esophagus detected on barium oesophograms: increased prevalence in patient with esophageal carcinoma. Am J Roentgenol 1995; 165: $1381-1385$

3 Teraishi F, Fujiwara T, Jikuhara A et al. Esophageal intramural pseudodiverticulosis with esophageal strictures successfully treated with dilation therapy. 2006; 82: 1119-1121
Bibliography

DOI $10.1055 / \mathrm{s}-2007-966595$

Endoscopy 2007; 39: E265-E266

(c) Georg Thieme Verlag KG Stuttgart · New York . ISSN 0013-726X

\section{Corresponding author}

\section{E. Wang, MD}

Department of General Surgery,

National University Hospital of Singapore

5 Lower Kent Ridge Road

Singapore 119074

Fax: +65-67722418

etiennewang@gmail.com 